\title{
Classification of poplar trees with object-based ensemble learning algorithms using Sentinel-2A imagery
}

https://doi.org/10.1515/jogs-2020-0003

Received January 24, 2020; accepted March 16, 2020

\begin{abstract}
The poplar species in the forest ecosystems are one of the most valuable and beneficial species for the society and environment. Conventional methods require high cost, time and labor need, and the results obtained vary and are insufficient in terms of achieved accuracy level. Determination of poplar cultivated fields and mapping of their spatial sites play a vital role for decisionmakers and planners to enhance the economic and ecological value of poplar trees. The study aims to map Poplar (P.deltoides) cultivated areas in Akyazi district of Sakarya, Turkey province using various combinations of the Sentinel-2A image bands. For this purpose, objectbased classification based on multi-resolution segmentation algorithm was utilized to produce image objects and ensemble learning algorithms, namely, Adaboost (AdaB), Random Forest (RF), Rotation Forest (RotFor) and Canonical correlation forest (CCF) were applied to produce thematic maps. In order to analyze the effects of the spectral bands of the Sentinel-2A image on the object-based classification performance, three datasets consisting of different spectral band combinations (i.e. four $10 \mathrm{~m}$ bands, six $20 \mathrm{~m}$ bands and ten $10 \mathrm{~m}$ pan-sharpened bands) were used. The results showed that the RotFor and CCF classifiers produced superior classification performances compared to the AdaB and RF classifiers for the band combinations regarded in this study. Moreover, it was found that determination of poplar tree class level accuracy reached to $94 \%$ in terms of F-score. It was also observed that the inclusion of the six spectral bands at $20 \mathrm{~m}$ resolution resulted in a noteworthy increase in classification accuracy (up to 6\%) compared to single $10 \mathrm{~m}$ band combination.
\end{abstract}

Keywords: Ensemble Learning, Poplar Trees, Object-based Image Analysis, Sentinel-2A

H. Tonbul, I. Colkesen: Gebze Technical University, Department of Geomatics Engineering, Gebze-Kocaeli, Turkey,

E-mail: htonbul@gtu.edu.tr, icolkesen@gtu.edu.tr

\section{Introduction}

Remote sensing technologies provide vital information for the visualization, processing and analyzing of the Earth observation data. Determination of land use and land cover (LULC) types of the Earth's surface and production of thematic maps derived from the remotely sensed imagery using a particular image classification technique has been one of the most concentrated topics in remote sensing field. These thematic maps are mainly used for forestry, agriculture, geology, natural resource, change detection and land management plans (Kavzoglu and Colkesen, 2013; Machala and Zejdová, 2014; Colkesen et al., 2016). There are many studies that have been carried out for the determination of cultivated crops and tree species, also monitoring cultivated croplands, which are of great importance in terms of sustainable environment and ecological balance (Danielson et al., 2016; Immitzer et al., 2016; Colkesen and Kavzoglu, 2017a).

Poplar trees are among the most valuable and beneficial species in the cultivated tree species. The Poplar trees have important characteristics including fast growth, ease of propagation and propensity to hybridize. Thanks to their useful features, as well as providing wood, fibre and other forest products, poplars provide benefits to society in the rehabilitation of degraded land, restoration of forest landscapes and mitigation of climate change (Isebrands and Richardson, 2014). There are more than 100 species of poplar trees, subspecies variability, and countless hybrids and clones located in the world. According to the latest National Poplar Commission Country Progress Reports covering the years 2012-2015, the area under poplar plantation in Turkey is about 145,000 hectares. Turkey is ranked the fourth in the world in terms of Poplar plantation size. The product obtained from these fields is ap-

\footnotetext{
^Corresponding Author: T. Kavzoglu: Gebze Technical University, Department of Geomatics Engineering, Gebze-Kocaeli, Turkey, E-mail: kavzoglu@gtu.edu.tr
} 
proximately 3.7 million $\mathrm{m}^{3}$ per year (Velioglu and Akgül, 2016). Determination of plantation coverage, gallery populus, field border and wind curtain areas registered with certified populus clones is important for national and international forestry sector.

Determination of poplar cultivated areas from the remotely sensed imagery could be cost and time-effective compared to traditional terrestrial methods for decision makers and planners. In the literature, existing satellite sources, such as MODIS, Landsat or SPOT data has been already used in different cropland mapping due to their global coverages (Yan and Roy, 2015; Colkesen and Kavzoglu, 2017a; Qiu et al., 2017). Since its launch, Sentinel-2, an open-source optical remote sensing system, has gained popularity in remote sensing community for cropland monitoring and mapping, thanks to its high spatial resolution and high temporal resolution. With high spatial resolution, Sentinel-2A data provides the possibility of cropland mapping by applying the object-based image analysis.

Classification of remotely sensed imagery is the most effective way for producing the LULC maps. Accuracy and reliability of these maps is prerequisite for success of the further applications at local and global scales. For this purpose, variety of classification approaches (i.e. pixel, sub-pixel and object-based) and classification algorithms (i.e. parametric and non-parametric classifiers) have been suggested and implemented in the literature (Mather and Koch, 2011). In recent years, the popularity of using machine learning methods has been increased in the the classification of remote sensing imagery (Wu et al. 2016; Colkesen and Kavzoglu, 2017a; Kavzoglu et al., 2018; Sahin et al., 2018). Within the machine learning concept, ensemble learning algorithms based on the aggregation of the individual predictions of multiple weak learners to make a final decision on a given classification problem have been widely preferred by researchers due to their robustness and accuracy in thematic mapping (Colkesen and Kavzoglu, 2017a; Kenduiywo et al., 2018; Sonobe et al., 2018). Previous studies have reported that classification performance of the decision tree-based ensemble learning algorithms such as bagging, Adaboost (AdaB), random forest $(\mathrm{RF})$, rotation forest (RoF) and recently introduced canonical correlation forest (CCF) was superior compared to traditional classifiers (Gislason et al., 2006; Kavzoglu and Colkesen, 2013; Kavzoglu et al., 2015; Li et al., 2016; Xiu et al., 2017; Sahin et al., 2018).

The main objective of this paper is to map Poplar (P.deltoides) cultivated areas from a diverse agricultural landscape situated in Turkey using a Sentinel-2A satellite image. For this purpose, object-based image classification with four popular ensemble learning algorithms, namely, AdaB, RF, RotFor and CCF were performed to produce thematic map of the study site. Furthermore, in order to estimate the effect of $10 \mathrm{~m}$ and $20 \mathrm{~m}$ spectral bands of the Sentinel-2A imagery on classification accuracy, three different datasets including $10 \mathrm{~m}, 20 \mathrm{~m}$ and fused bands of the image were taken into consideration to perform objectbased image classification.

\section{Study Area and Dataset}

The study area is located in the north-western part of Turkey (Fig. 1) and it covers approximately $560 \mathrm{~km}^{2}$ agricultural lands. Agriculture is the main part of the region's economy, especially with the production of wheat, sugar beet, corn and hazelnut. In addition, the study area is one of the suitable sites for cultivating hybrid poplar trees in Turkey. The study area is composed of five main LULC classes; (1) forest class including coniferous and deciduous trees, (2) cultivated class including areas containing agricultural products that are not harvested, (3) noncultivated class including harvested or uncultivated lands, (4) poplar class including hybrid poplar cultivated lands and (5) urban class including impervious surfaces such as main roads, residential and industrial buildings.

The selection of appropriate remotely sensed data is of great importance in obtaining a successful classification result. In general, a classification system is mainly determined based on user needs, spatial resolution of remotely sensed data, classification algorithms and time constraints (Şatir and Berberoglu, 2012). Remote sensing availability in the production of forest cover and species maps has become more common since the 1970s (Satir et al., 2017). In order to produce the LULC maps of the study area, Sentinel-2 imagery, a main product of the state-ofart sensor launched 2015 by European Space Agency, was used as a main data source in this study. In recent years, there has been an increasing interest in the use of Sentinel2 imagery providing high spatial and spectral resolution (i.e. four $10 \mathrm{~m}$ and six $20 \mathrm{~m}$ bands) for tree species classification problems (Persson et al. 2018; Grabska et al. 2019). A cloud-free Sentinel-2 multispectral image acquired on October 22, 2016, retrieved from Copernicus Open Access Hub, covering the bulk of the study area was used as a main data source in this study. It should be noted that in order to minimize the misclassification rate between the spectrally similar classes (i.e. agricultural products, forest and poplar trees), the image acquired on October was utilized in this study. 


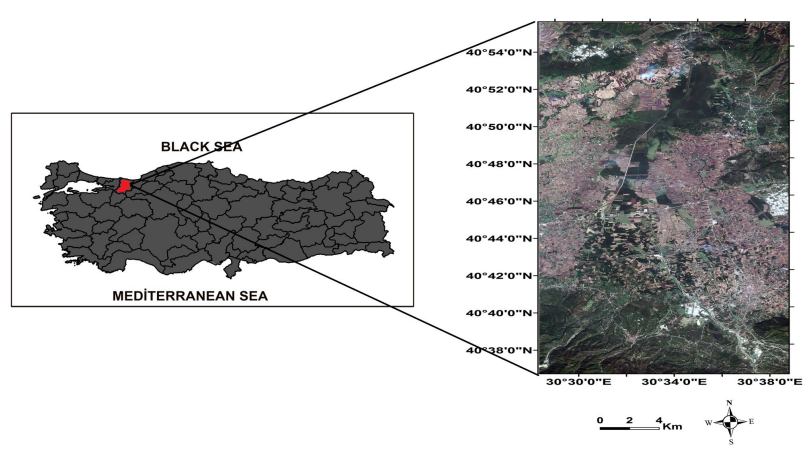

Figure 1: The location of the study site.

In accordance with the purpose of the study, three datasets comprising different spectral band combinations were formed and utilized for the LULC classification. The first dataset, thereafter called as Dataset-1, comprises the original $10 \mathrm{~m}$ resolution bands of the Sentinel-2A imagery (i.e. band 2,3,4 and 8). The second dataset, named as Dataset-2, contains original $20 \mathrm{~m}$ resolution bands of the Sentinel image (i.e. band 5, 6, 7, 8A, 11 and 12). The third dataset, named as Dataset-3, contains both at $10 \mathrm{~m}$ resolution and six pansharpened $20 \mathrm{~m}$ spectral bands of imagery (totally ten bands). It should be noted that the GramSchmidt spectral sharpening algorithm was utilized to spatially resample the $20 \mathrm{~m}$ resolution bands to $10 \mathrm{~m}$ using the pan-sharpening procedure suggested by Selva et al. (2015). All pre-processing and pan-sharpening analyses were carried out in ENVI software (v.5.1). The spectral bands and their spatial resolutions of the Sentinel-2A image and three datasets considered in this study are given in Table 1. It should be noted that while $\checkmark$ symbol indicates the spectral band is included in the current dataset, $x$ symbol indicates vice-versa. As can be seen from the Table specific bands of coastal aerosol, water vapour and cirrus are not considered in this study.

In this study, OBIA based multi-resolution segmentation, which available in eCognition software was employed for all segmentation processes. It should be noted that all spectral bands were equally weighted.

\section{Methodology}

In this study, the poplar tree cultivated lands were determined from the Sentinel imagery using object-based image classification with three ensemble learning algorithms. In this context, fundamental processing steps of object-based supervised image classification including creation of image objects (i.e. segmentation), determination of image object features, selection of training and validation samples, classification of segmented image using ensemble learning algorithms and accuracy assessment were employed, respectively (Fig. 2). It should be noted that available forestry maps, fine-resolution images from Google Earth and field surveys carried out using a handheld GPS were used to create ground reference data.

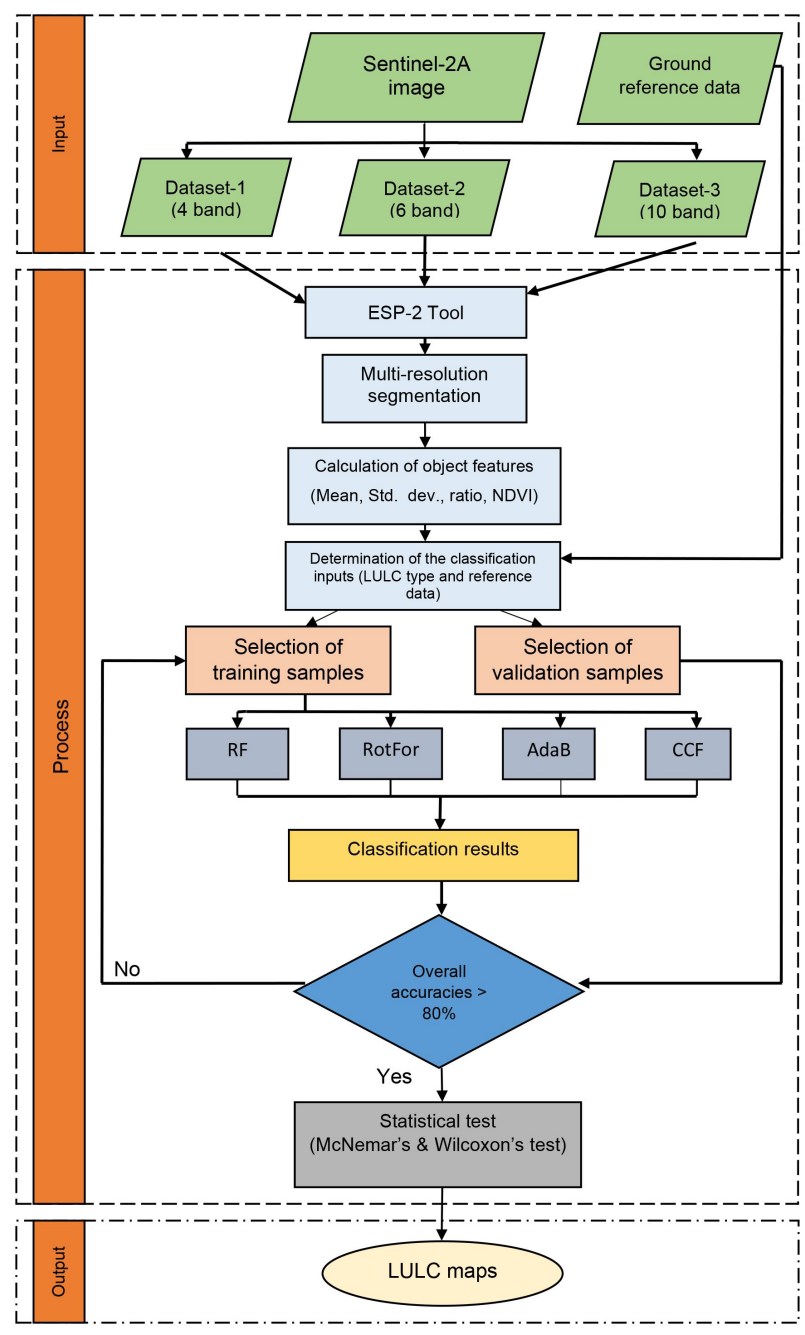

Figure 2: Flowchart of methodology performed in this study.

\subsection{OBIA Based Image Segmentation}

The first and the most significant processing step of objectbased image analysis is image segmentation. In this paper, the multi-resolution segmentation algorithm proposed by Baatz and Schäpe (2000) as a region growing method was used to create segmented image objects. The algorithm starts from the pixel level and groups pixels using local 
Table 1: Sentinel-2A satellite sensor specifications and details of dataset used in this study.

\begin{tabular}{|c|c|c|c|c|c|}
\hline Band & Band Name & $\begin{array}{c}\text { Spatial resolution } \\
(\mathrm{m})\end{array}$ & $\begin{array}{c}\text { Dataset- } \\
1 \\
\end{array}$ & $\begin{array}{c}\text { Dataset- } \\
2 \\
\end{array}$ & $\begin{array}{c}\text { Dataset- } \\
3 \\
\end{array}$ \\
\hline 1 & Coastal aerosol & 60 & $x$ & $x$ & $\mathrm{x}$ \\
\hline 2 & Blue & 10 & $\checkmark$ & $x$ & $\checkmark$ \\
\hline 3 & Green & 10 & $\checkmark$ & $x$ & $\checkmark$ \\
\hline 4 & Red & 10 & $\checkmark$ & $x$ & $\checkmark$ \\
\hline 5 & $\begin{array}{l}\text { Vegetation red } \\
\text { edge } \\
\text { Vegetation red }\end{array}$ & 20 & $x$ & $\checkmark$ & $\checkmark$ \\
\hline 6 & edge & 20 & $x$ & $\checkmark$ & $\checkmark$ \\
\hline 7 & edge & 20 & $x$ & $\checkmark$ & $\checkmark$ \\
\hline 8 & NIR & 10 & $\checkmark$ & $\mathrm{x}$ & $\checkmark$ \\
\hline $8 \mathrm{~A}$ & Narrow NIR & 20 & $\mathrm{x}$ & $\checkmark$ & $\checkmark$ \\
\hline 9 & Water vapour & 60 & $\mathrm{x}$ & $\mathrm{x}$ & $x$ \\
\hline 10 & SWIR - Cirrus & 60 & $x$ & $x$ & $x$ \\
\hline 11 & SWIR & 20 & $x$ & $\checkmark$ & $\checkmark$ \\
\hline 12 & SWIR & 20 & $x$ & $\checkmark$ & $\checkmark$ \\
\hline
\end{tabular}

homogeneity criteria. It comprises three user-selected parameters namely, scale, shape and compactness. The scale parameter is the most crucial parameter that adjusts the control average image object size. The higher value determined for the scale parameter, the larger image objects acquired (Kavzoglu et al., 2017; Kavzoglu and Tonbul, 2018). In the literature, a trial-and-error strategy relied on experience of operator is one of the suggested methods to determine the optimal scale parameter (Witharana and Civco, 2014; Ma, 2015). Since user knowledge and experience is important issue for optimal parameter selection, performing this strategy can be difficult and time consuming (Belgiu and Drăgut, 2014; Johnson et al., 2015; Kavzoglu et al., 2017). In order to determine the appropriate scale parameter, the ESP-2 tool (Estimation of Scale Parameter 2) developed by Drăgut et al. (2014) was employed in this study. The tool selects optimal scales using the local variance of the image and the rate of change values of local variance was used. It was programmed in Cognition Network Language and has been widely used in many studies (Belgiu and Drăgut, 2014; Kavzoglu et al., 2017; Kavzoglu and Tonbul, 2018).

\subsection{Ensemble Learning Algorithms}

In this study, four decision-tree (DT) based ensemble learning algorithms namely, AdaBoost (AdaB), random forest (RF), rotation forest (RotFor), and canonical correlation forest (CCF) were utilized to classify segmented image segments with respect to their features.
Adaptive Boosting or widely named as AdaB is introduced by Freund and Schapire (1997) as a member of boosting family of ensemble learning algorithm. The main idea behind the AdaB is to create an ensemble model incrementally, adding one classifier at a time (Kuncheva, 2014). In each iteration, training data are formed depending on the classification performance of previous classifier. In other words, after the first iteration, misclassified samples are used more often compared to correctly classified samples in each iteration. Thus, the algorithm constructs a next classifier that intends to correct errors in the previous iteration (Kim, 2009; Kavzoglu and Colkesen, 2013).

The RF algorithm proposed by Breiman (2001) as an ensemble learning technique has been widely used for many classification problems due to their accuracy and robustness (Belgiu and Drăguţ, 2016). The algorithm also known as decision forest due to it uses the DT classifier as the base classifier in the ensemble. As the name implies, the RF algorithm uses a set of DT classifiers to construct an ensemble model and it combines their individual predictions by applying majority voting rule to make a final prediction on an unknown sample (Rokach, 2010). Since the DT is used as the base classifier, in order to ensure diversity in the ensemble model, different training samples formed by applying the bootstrap aggregation strategy from the original input dataset are used in the construction stage of individual DT classifiers. In each DT construction stage, about $2 / 3$ of the bootstrapped samples (i.e. in-bag samples) are used to train the DT and the rest (i.e. 
out-of-the bag samples) are used to estimate classification error (Breiman, 2001; Kavzoglu, 2007).

The RotFor is introduced by Rodriguez et al. (2006) as a variant of the $R F$ classifier. The main difference between the $\mathrm{RF}$ is that before the DT construction stage, to ensure extra diversity, the RotFor algorithm first applies orthogonal transformation technique (i.e. principal component analysis) to bootstrapped samples and then transformed components based on a similar principle to RF (Kavzoglu and Colkesen, 2013; Kavzoglu et al., 2015). The main purpose of RotFor is to first apply principal component analysis to the bagged samples and then to construct a decision tree based on the resulting components. Thus, it is possible to increase individual accuracy of an ensemble classifier by promoting extra diversity. (Zhang and Zhang, 2010).

More recently, the CCF algorithm is proposed by Rainforth and Wood (2015) as an improved version of the RF algorithm. The main heuristic behind the CCF is to apply canonical correlation analysis to the bootstrapped samples before the DT construction phase. Since each DT in the ensemble model is trained with canonical correlation components, they are named as canonical correlation trees that are oblique decision trees. Unlike the PCA, the canonical correlation analysis is applied to it seek feature projections ensuring the maximum correlation between bands and the target classes (Rainforth and Wood, 2015; Colkesen and Kavzoglu, 2017a).

\subsection{Accuracy Assessment and Statistical Testing}

In this study, classification results were analyzed using standard accuracy measure named as overall accuracy. In addition, F-score measures based on the calculation of harmonic mean of precision (i.e. Producer's accuracy) and recall (i.e. User's accuracy) were also estimated to evaluate class-based accuracy. For further evaluation of the estimated results, two non-parametric statistical tests (i.e., McNemar's and Wilcoxon's Signed-Ranks tests) often required to evaluate the statistical significance of differences in predictions between the algorithms.

In the literature, McNemar's test and Wilcoxon's Signed-Ranks test have been widely used for this purpose (Demsar, 2006; Foody, 2009; Kavzoglu and Colkesen, 2013; Colkesen and Kavzoglu, 2017b). The McNemar's test is a non-parametric test, which is based on $\chi^{2}$ distribution calculation, generally performed to compare the classification errors of two classifiers. The second test is Wilcoxon's signed-rank test is a non-parametric statistical hypothesis test, based on sorting the performance differences of the two classification algorithms, sorting the calculated differences separately as positive and negative differences and then comparing them with each other (Kavzoglu et al., 2015). It should be noted that both statistical tests were two-tailed, and results were interpreted at $95 \%$ confidence interval.

\section{Results}

In order to estimate an optimal segmentation scale for the multi-resolution segmentation process, the ESP-2 tool was applied for all three datasets (Fig. 3). In addition to spectral bands, some features must also consider improving the quality of prediction, and thus classification accuracy.
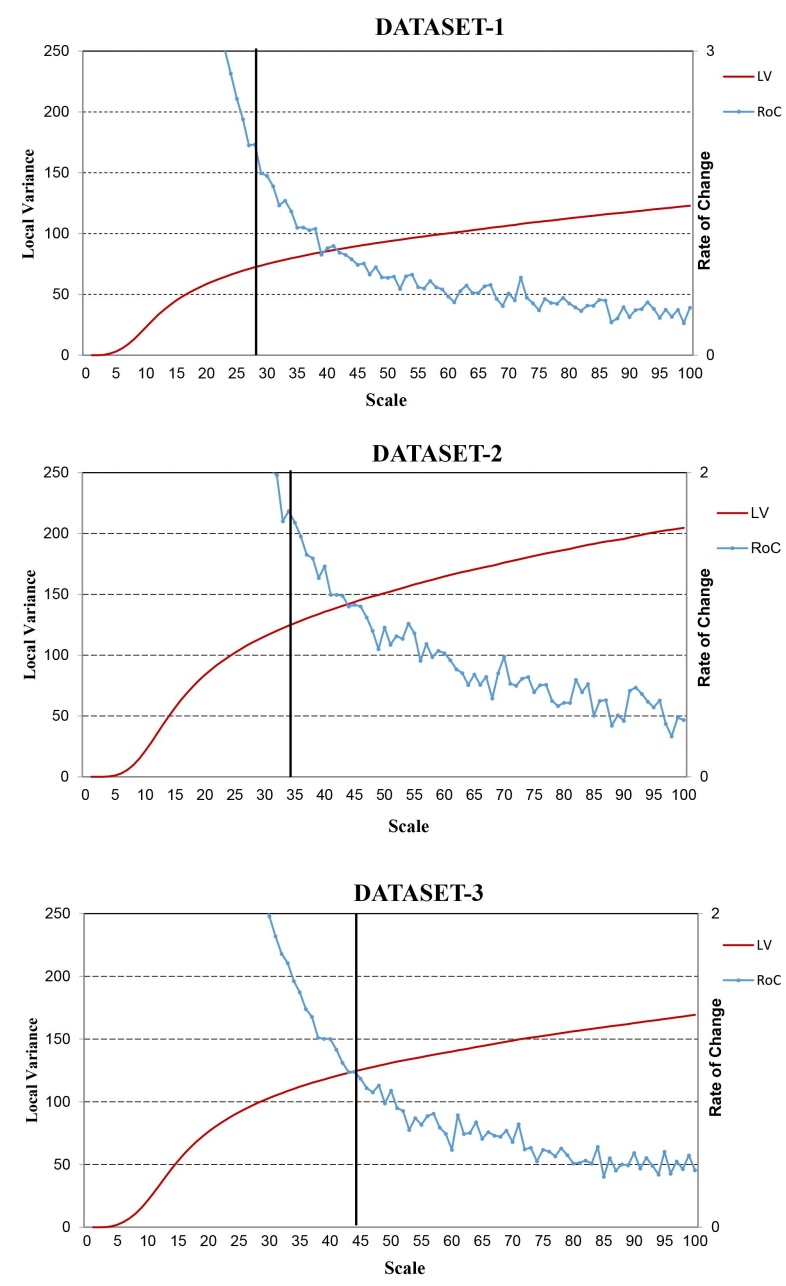

Figure 3: Optimal scale parameter selection using ESP-2 tool for all datasets. 
As shown in Fig. 3, scale levels of 28, 34 and 44 were estimated for Dataset 1, 2 and 3, respectively. Furthermore, the parameters of shape and compactness were set to the default values (i.e. 0.1 and 0.5, respectively) as used in many studies (Marpu et al., 2010; Kavzoglu et al., 2017). As a result of the segmentation process, totally 119,$433 ; 39,650$ and 87,566 image objects were produced for the datasets.

In order to classify segmented image objects, AdaB, $\mathrm{RF}$, RotFor and CCF classifiers were utilized using Matlab software. It should be noted that the mean, standard deviation of each spectral band, ratio of each spectral bands relative to each other (i.e., each band divided by the summation of all bands ranging from 0 to 1) and NDVI of Sentinel-2A image were considered as image object features in all classification processes for all datasets. The classification accuracies were calculated from standard confusion matrices using 1,000 pixels per class for all datasets. The estimated overall accuracies and F-score values of ensemble learners for all three data sets were given in Table 2. It was observed that the CCF classifier produced the highest overall classification accuracy (95.42\%) for Dataset-1 and Dataset-3 (95.80\%), whilst RotFor classifier yield the highest overall classification accuracy (92.78\%) for Dataset-2, however the difference was very small compared with the CCF result $(92.60 \%)$. On the other hand, the lowest overall accuracies were estimated with the RF classifier for Dataset-1 (89.44\%), Dataset-2 (88.74\%) and Dataset-3 (90.04\%). Results noticeably indicated that the RotFor and CCF classifiers outperformed the AdaB and RF algorithms for all datasets and the improvement in accuracy reached to $\sim 6 \%$ for Dataset-1, $\sim 4 \%$ for Dataset-2 and $\sim 5 \%$ for Dataset-3. Another important finding is that analysis of the individual class accuracies showed that the highest F-score value was calculated as $98.85 \%$ by RotFor classifier for cultivated class in Dataset-3. However, the lowest F-score value was estimated as $74.75 \%$ by the RF classifier for poplar tree class in Dataset-1. Furthermore, the highest F-score value for poplar tree class was computed as $93.94 \%$ by CCF classifier for Dataset-3 including original $10 \mathrm{~m}$ and pansharpened $20 \mathrm{~m}$ bands.

In order to statistically compare the classification performances, two non-parametric tests, namely, McNemar's and Wilcoxon's signed-ranks tests were employed (Table 3). Both McNemar's and Wilcoxon's signed-rank tests confirmed that differences in classification performances were statistically significant for all datasets pairwise comparison except for RotFor and CCF classifiers for Dataset-2 $\left(\chi_{0.05}^{2}=0.44\right.$ and $\left.z_{0.05}=0.74\right)$. The difference in classifier performances was only $0.18 \%$ in terms of overall accuracy.

In order to visually compared the classification results, the produced thematic maps with all classifiers were also given in Fig. 4.

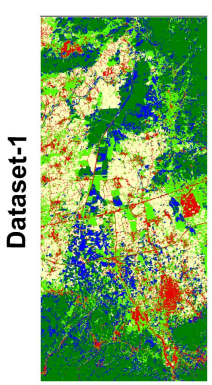

(a)

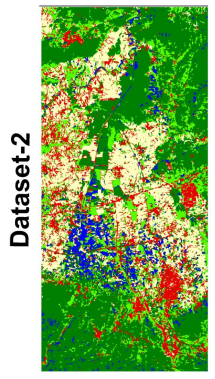

(a)

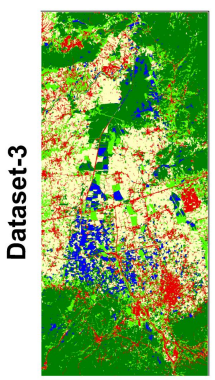

(a)

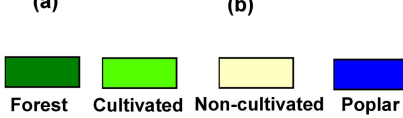

Forest Cultivated Non-cultivated Poplar

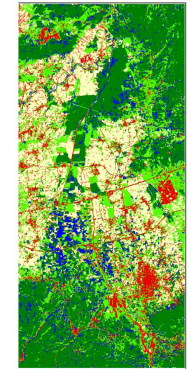

(b)

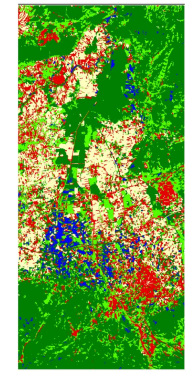

(b)

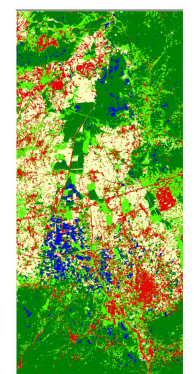

(b) (c)

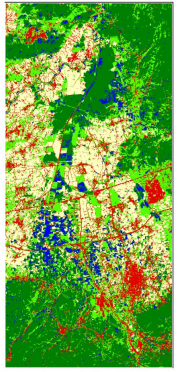

(c)

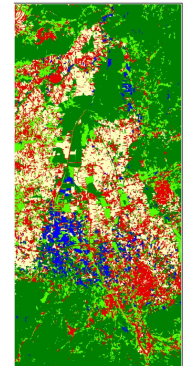

(c)

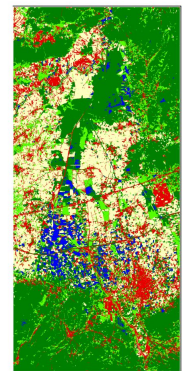

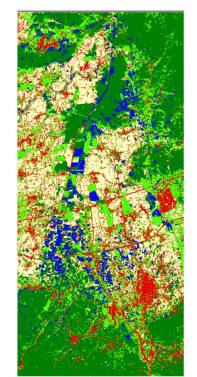

(d)

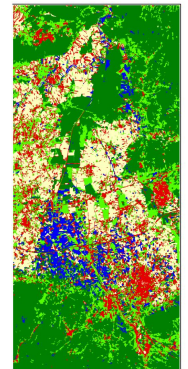

(d)

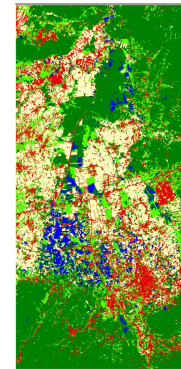

(d)

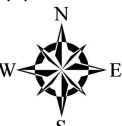

Figure 4: Thematic maps of the study site created by (a) AdaB, (b) RF, (c) RotFor and (d) CCF for all datasets.

Thematic maps produced for the test site revealed some important findings. It was observed that CCF and RotFor classifier produced similar results since they assigned the objects mostly to the same LULC categories for all datasets. Visual analysis of the thematic maps also revealed that it is difficult to discriminate class boundaries between poplar, cultivated and forest classes due to the misclassifications mainly resulted from the identification of spectrally similar classes (i.e. salt-and-pepper noise) in some regions. Furthermore, it was observed that high poplar tree areas generally situated in south part of the study site. 
Table 2: Classification Accuracy of Datasets.

\begin{tabular}{|c|c|c|c|c|c|c|c|c|c|c|c|c|}
\hline \multirow[t]{2}{*}{ LULC Class } & \multicolumn{3}{|c|}{ AdaB } & \multicolumn{3}{|c|}{$\begin{array}{c}\text { RF } \\
\text { F-score (\%) }\end{array}$} & \multicolumn{3}{|c|}{$\begin{array}{c}\text { RotFor } \\
\text { F-score (\%) }\end{array}$} & \multicolumn{3}{|c|}{$\begin{array}{c}\text { CCF } \\
\text { F-score (\%) }\end{array}$} \\
\hline & DT-1 & DT-2 & DT-3 & DT-1 & DT-2 & DT-3 & DT-1 & DT-2 & DT-3 & DT-1 & DT-2 & DT-3 \\
\hline Forest & 89.28 & 83.35 & 92.39 & 80.92 & 84.43 & 82.36 & 89.54 & 88.59 & 91.34 & 92.70 & 87.78 & 93.89 \\
\hline Cultivated & 96.78 & 93.11 & 98.50 & 96.88 & 93.59 & 98.11 & 97.12 & 98.41 & 98.85 & 98.15 & 97.60 & 98.65 \\
\hline Non-cultivated & 97.21 & 95.99 & 94.63 & 97.25 & 95.21 & 95.97 & 98.43 & 95.73 & 97.64 & 97.33 & 97.41 & 96.53 \\
\hline Poplar & 88.36 & 78.86 & 92.21 & 74.75 & 76.53 & 77.62 & 89.20 & 85.96 & 90.36 & 92.03 & 83.04 & 93.94 \\
\hline Urban & 96.61 & 94.83 & 94.46 & 96.58 & 93.44 & 95.55 & 98.07 & 94.91 & 97.34 & 96.86 & 96.71 & 96.00 \\
\hline $\mathrm{OA}(\%)$ & 93.64 & 89.24 & 94.44 & 89.44 & 88.74 & 90.04 & 94.46 & 92.78 & 95.12 & 95.42 & 92.60 & 95.80 \\
\hline
\end{tabular}

OA: Overall Accuracy; DT-1: Dataset-1; DT-2: Dataset-2; DT-3: Dataset-3

Table 3: Statistical Comparison of Classifier Performances.

\begin{tabular}{|c|c|c|c|c|c|c|}
\hline \multirow[b]{2}{*}{ Pairwise Comparison } & \multicolumn{2}{|c|}{ DT-1 } & \multicolumn{2}{|c|}{ DT-2 } & \multicolumn{2}{|c|}{ DT-3 } \\
\hline & McNemar's & Wilcoxon's & McNemar's & Wilcoxon's & McNemar's & Wilcoxon's \\
\hline RF vs RotFor & 219.30 & 14.87 & 185.33 & 13.68 & 237.07 & 15.46 \\
\hline AdaB vs RF & 137.36 & 11.78 & 6.47 & 2.65 & 162.03 & 12.79 \\
\hline AdaB vs RotFor & 7.18 & 2.74 & 142.75 & 12.02 & 5.04 & 2.31 \\
\hline RF vs CCF & 225.96 & 15.08 & 162.40 & 12.81 & 252.67 & 15.95 \\
\hline RotFor vs CCF & 11.05 & 3.39 & 0.44 & 0.74 & 8.25 & 2.96 \\
\hline AdaB vs CCF & 25.73 & 5.13 & 115.24 & 10.80 & 32.53 & 5.79 \\
\hline
\end{tabular}

Note that test values $>3.84$ show that statistical difference at 95\% confidence level for McNemar's test and test values $>1.96$ indicate statistical difference at 95\% confidence level for Wilcoxon's signed-rank test. Bold values show calculated statistics smaller than the critical values $\left(\chi_{0.05}^{2}\right)=3.84$ and $\left.z_{0.05}=1.96\right)$.

\section{Conclusion}

The purpose of this study was to delineate poplar tree areas using four ensemble learning algorithms, namely AdaB, RF, RotFor, and CCF classifiers using a Sentinel-2A imagery. In this context, OBIA-based multi-resolution segmentation method was applied for the segmentation process. Furthermore, three datasets involve different spectral band combinations, namely Dataset-1, Dataset-2 and Dataset-3 were processed to evaluate the effect of spectral band combinations on classification performance.

The following conclusions can be drawn from the results produced in this study. First, the results noticeably showed that CCF and RotFor classifiers outperformed the AdaB and RF classifiers for all cases and improvements in classification performance reached to approximately $6 \%$ in terms of overall accuracy. The findings also underlined that the CCF classifier performed best overall accuracy results for Dataset-1 and Dataset-3, whilst RotFor classifier performed best overall accuracy results for Dataset-2. However the difference was negligible with CCF classifier. The key advantages of CCF can be expressed as the use of less ensemble learners (i.e. trees) and less sensitivity to param- eter setting from the user part as stated by Colkesen and Kavzoglu (2017a). Furthermore, superiority of RotFor over $\mathrm{RF}$ might be related to training approach of RotFor, which is trained on whole dataset in a rotated feature space generated from PCA transformation. Second, it was observed that the overall accuracy of Dataset-3 outperformed the Dataset-1 and Dataset-2 overall accuracies for all classifiers. To be more specific, the highest classification accuracy was estimated as $95.80 \%$ for Dataset-3, whilst the highest classification accuracy was computed as $95.42 \%$ and $92.78 \%$ for Dataset- 1 and Dataset-2, respectively. This could be result of the better identification between the pixels by means of additional spectral information in $20 \mathrm{~m}$ SWIR and NIR bands of Sentinel-2A imagery. This result also clearly indicate that the use of additional bands at $20 \mathrm{~m}$ resolution of Sentinel-2A image provided remarkable information for better delineation of the LULC classes and classification of agricultural crops. Furthermore, Dataset1 classification accuracies have outperformed to Dataset2 classification accuracies for all classification algorithms related to better spectral band resolution (i.e. $10 \mathrm{~m}$ vs $20 \mathrm{~m}$ ). Third, the both statistical test (i.e., McNemar's and Wilcoxon's signed-rank test) results confirmed that the accuracy differences were estimated statistically signifi- 
cant for all cases except for RotFor and CCF classifier for Dataset-2. These findings also showed that McNemar's and Wilcoxon's signed-rank statistical tests performed parallel results for pairwise comparison. Finally, when the produced maps were analyzed, it was seen that high poplar tree density sites were mostly situated in the south region of the study site.

\section{References}

Baatz, M. and Schäpe A. 2000, Multi resolution segmentation-an optimization approach for high quality multi scale image seg. mentation. In: Strobl, J., Blaschke, T., Griesebner, G. (Ed.), Angewandte Geographische Informations verarbeitung XII; Karlsruhe, Germany, 12-23

Belgiu M. and Drăgut L., 2014, Comparing supervised and unsupervised multiresolution segmentation approaches for extracting buildings from very high resolution imagery. ISPRS J. Photogramm. Remote Sens., 9, 6, 67-75, doi: 10.1016/j.isprsjprs.2014.07.002

Belgiu M. and Drăguţ L., 2016. Random forest in remote sensing: A review of applications and future directions, ISPRS J. Photogramm. Remote Sens., 114, 24-31, doi: 10.1016/j.isprsjprs.2016.01.011

Breiman L., 2001, Random Forests. Mach. Learn., 45(1), 5-32, doi: 10.1023/A:1010933404324

Colkesen I., Sahin E.K. and Kavzoglu T., 2016, Susceptibility mapping of shallow landslides using kernel-based Gaussian process, support vector machines and logistic regression. J. Afr. Earth Sci., 118, 53-64, doi: 10.1016/j.jafrearsci.2016.02.019

Colkesen I. and Kavzoglu T., 2017a, Ensemble-based canonical correlation forest (CCF) for land use and land cover classification using Sentinel-2 and Landsat OLI imagery. Remote Sens. Lett., 8, 10821091, doi: 10.1080/2150704X.2017.1354262

Colkesen I. and Kavzoglu T., 2017b, The Use of Logistic Model Tree (LMT) for Pixel- and Object- Based Classifications Using HighResolution WorldView-2 Imagery. Geocarto Int., 32(1), 71-86, doi: 10.1080/10106049.2015.1128486

Danielson P., Yang L., Jin S., Homer C. and Napton D., 2016, An Assessment of the Cultivated Cropland Class of NLCD 2006 Using a Multi-Source and Multi-Criteria Approach. Remote Sens., 8(101), 1-16, doi: 10.3390/rs8020101

Drăgut L., Csillik O., Eisank C. and Tiede D., 2014, Automated parameterisation for multi-scale image segmentation on multiple layers. ISPRS J. Photogramm., 88, 119-127, doi: 10.1016/j.isprsjprs.2013.11.018

Demsar J., 2006, Statistical comparisons of classifiers over multiple data sets. J. Mach. Learn. Res., 7, 1-30

Foody G.M., 2009, Classification accuracy comparison: hypothesis tests and the use of confidence intervals in evaluations of difference, equivalence and non-inferiority. Remote Sens. Environ., 113, 1658-1663, doi: 10.1016/j.rse.2009.03.014

Freund Y. and Schapire RE. 1997, A decision-theoretic generalization of on-line learning and an application to boosting. J Comput Syst Sci., 55, 119-139, doi: 10.1006/jcss.1997.1504

Gislason P. O., Benediktsson J.A. and Sveinsson J. R., 2006, Random Forests for Land Cover Classification. Pattern Recogn. Lett., 27 (4),
294-300, doi: 10.1016/j.patrec.2005.08.011

Grabska E., Hostert P., Pflugmacher D. and Ostapowicz, K., 2019, Forest Stand Species Mapping Using the Sentinel-2 Time Series. Remote Sens., 11(10), 1197, doi: 10.3390/rs11101197

Immitzer M., Vuolo F., Atzberger C., 2016, First experience with Sentinel-2 data for crop and tree species classifications in Central Europe. Remote Sens., 8(3), 166, doi: 10.3390/rs8030166

Isebrands J.G. and Richardson J., 2014. Poplars and Willows: Trees for Society and the Environment CAB International and Food and Agriculture Organization of the United Nations (FAO), CABI, p. 634.

Johnson B., Bragais M., Endo I., Magcale-Macandog D. and Macandog P., 2015. Image Segmentation Parameter Optimization considering Within- and Between-Segment Heterogeneity at Multiple Scale Levels: Test Case for Mapping Residential Areas Using Landsat Imagery. ISPRS Int. J. Geo-inf., 4, 2292-2305. doi:10.3390/ijgi4042292.

Kavzoglu T. and Colkesen I, 2013, An assessment of the effectiveness of a Rotation Forest ensemble for land-use and landcover mapping. Int. J. Remote Sens., 34 (12), 4224-4241, doi: 10.1080/01431161.2013.774099

Kavzoglu T., Colkesen I. and Yomralioglu T., 2015, Object-based classification with rotation forest ensemble learning algorithm using very-high-resolution WorldView-2 image. Remote Sens. Lett., 6(11), 834-843, doi: 10.1080/2150704X.2015.1084550

Kavzoglu T., 2017. Object-Oriented Random Forest for High Resolution Land Cover Mapping Using Quickbird- 2 Imagery. In: Samui P., Roy, S.S. Balas V.E. (Eds.), Handbook of Neural Computation, Elsevier. 607-619.

Kavzoglu T., Yildiz Erdemir M. and Tonbul H., 2017, Classification of semiurban landscapes from very highresolution satellite images using a regionalized multiscale segmentation approach. J Appl Remote Sens., 11(3), 035016, doi: 10.1117/1.JRS.11.035016

Kavzoglu T. and Tonbul H., 2018, An experimental comparison of Multi-Resolution Segmentation, SLIC and KMeans clustering for object-based classification of VHR imagery. Int. J. Remote Sens., 39(18), 6020-6036, doi: 10.1080/01431161.2018.1506592

Kavzoglu T., Tonbul H., Yildiz Erdemir M. and Colkesen I., 2018, Dimensionality reduction and classification of hyperspectral images using object-based image analysis, J Indian Soc. Remote., 46(8), 1297-1306 doi, 10.1007/s12524-018-0803-1.

Kenduiywo B.K., Bargiel D. and Soergel U., 2018, Crop-type mapping from a sequence of Sentinel 1 images. Int. J. Remote Sens., 39, 6383-6404, doi: 10.1080/01431161.2018.1460503

Kim, Y. S., 2009, Boosting and measuring the performance of ensembles for successful database marketing. Expert Syst Appl., 36, 2161-2176, doi: 10.1016/j.eswa.2007.12.036

Kuncheva L.I., 2014. Combining Pattern Classifiers: Methods and Algorithms, 2nd edition, John Wiley \& Sons Hoboken, New Jersey, 384p, doi: 10.1002/9781118914564

Li M., Ma L., Blaschke T., Cheng L. and Tiede D., 2016, A systematic comparison of different object-based classification techniques using high spatial resolution imagery in agricultural environments. Int. J. Appl. Earth Obs. Geoinf., 49, 87-98, doi: 10.1016/j.jag.2016.01.011

Ma L., 2015, Training set size, scale, and features in geographic object-based image analysis of very high resolution unmanned aerial vehicle imagery, ISPRS J. Photogramm. Remote Sens., 102, 14-27, doi: 10.1016/j.isprsjprs.2014.12.026

Machala M. and Zejdová L., 2014, Forest Mapping Through Objectbased Image Analysis of Multispectral and LiDAR Aerial Data. Eur J 
Remote Sens., 47, 117- 131, doi: 10.5721/EuJRS20144708

Marpu P.R., Neubert M., Herold H. and Niemeyer I., 2010, Enhanced evaluation of image segmentation results. J. Spat. Sci., 55(1), 55 68, doi: 10.1080/14498596.2010.487850

Mather P.M. and Koch M., 2011, Computer Processing of RemotelySensed Images: An Introduction. 4th Edition, Chichester, UK: Wiley-Blackwell, 434p, doi: doi.org/10.1002/9780470666517

Persson M., Lindberg E. and Reese, H., 2018, Tree Species Classification with Multi-Temporal Sentinel-2 Data. Remote Sens., 10,1794, doi: $10.3390 /$ rs10111794

Qiu B., Luo Y., Tang Z., Chen C., Lu D., Huang H., Chen Y., Chen N. and Xu W., 2017, Winter wheat mapping combining variations before and after estimated heading dates. ISPRS J. Photogramm. Remote Sens., 123, 35-46, doi: 10.1016/j.isprsjprs.2016.09.016

Rainforth T. and Wood F. Canonical Correlation Forests. 2015, https://arxiv.org/pdf/1507.05444.pdf. Accessed 26 December 2019

Rodriguez J.J., Kuncheva L. and Alanso, C. J., 2006, Rotation Forest: A New Classifier Ensemble Method. IEEE T Pattern Anal., 28 (10), 1619-1630, doi: 10.1109/TPAMI.2006.211

Rokach L., 2010, Ensemble-Based Classifiers. Artif. Intell. Rev., 33(12), 1-39. doi:10.1007/s10462-009-9124-7

Sahin E.K., Colkesen I. and Kavzoglu T., 2018, A comparative assessment of canonical correlation forest, random forest, rotation forest and logistic regression methods for landslide susceptibility mapping. Geocarto Int., 1-23, doi: 10.1080/10106049.2018.1516248

Satir O., Berberoglu S., Akca E. and Yeler O., 2017, Mapping the Dominant Forest Tree Distribution Using Combined Image Classification Approach in a Complex Eastern Mediterranean Basin. J. Spat.Sci., 62, 157-171, doi: 10.1080/14498596.2016.1212414

Selva M., Aiazzi B., Butera F., Chiarantini L. and Baronti S., 2015, Hyper-Sharpening: A First Approach on SIM-GA Data. IEEE J-Stars., 8 (6), 3008-3024, doi: 10.1109/JSTARS.2015.2440092
Sonobe R., Yamaya Y., Tani H., Wang X., Kobayashi N. and Mochizuki K.-I., 2018, Crop classification from Sentinel-2-derived vegetation indices using ensemble learning. J.Appl. Remote Sens., 12(2), 026019, doi: 10.1117/1.JRS.12.026019

Şatir O. and Berberoglu S., 2012, Land Use/Cover Classification Techniques Using Optical Remotely Sensed Data in Landscape Planning, In: Murat Özyavuz (Ed.), Landscape Planning, Intech, Rijeka, 21-54.

Velioglu E. and Akgül S., 2016, Poplars and willows in Turkey: country progress report of the national poplar commision time period: 2012-2015, poplar and fast growing forest trees. Research Institute, İzmit/Turkey

Witharana C. and Civco D. L., 2014, Optimizing multi-resolution segmentation scale using empirical methods: exploring the sensitivity of the supervised discrepancy measure Euclidean distance 2 (ED2), ISPRS J. Photogramm. Remote Sens., 87, 108-121, doi: 10.1016/j.isprsjprs.2013.11.006

Wu C., Shen H., Shen A., Deng J., Gan M., Zhu J., Hongwei X. and Wang K., 2016, Comparison of Machine-Learning Methods for AboveGround Biomass Estimation Based on Landsat Imagery. J Appl Remote Sens., 10(3), 035010, doi: 10.1117/1.JRS.10.035010

Xiu Y., Liu, W. and Yang W., 2017, An Improved Rotation Forest for Multi-Feature Remote-Sensing Imagery Classification. Remote Sens., 9, 1205, doi: 10.3390/rs9111205

Yan L. and Roy D.P., 2015, Improved time series land cover classification by missing-observation-adaptive nonlinear dimensionality reduction. Remote Sens. Environ., 158, 478-491, doi: 10.1016/j.rse.2014.11.024

Zhang C.-X. and Zhang J.-S., 2010, A Variant of Rotation Forest for Constructing Ensemble Classifiers. Pattern Anal Appl., 13(1), 5977, doi: 10.1007/s10044-009-0168-8 